\title{
SIRT6 inhibits growth of gastric cancer by inhibiting JAK2/STAT3 pathway
}

\author{
JIANMEI ZHOU ${ }^{1}$, ANG WU ${ }^{1}$, XIAOTING YU ${ }^{2}$, JIANWEI ZHU ${ }^{1}$ and HUI DAI ${ }^{3}$ \\ ${ }^{1}$ Department of Medical Oncology, ${ }^{2}$ Intensive Care Unit, ${ }^{3}$ Department of Radiotherapy, \\ Hangzhou Cancer Hospital, Hangzhou, Zhejiang 310002, P.R. China
}

Received January 24, 2017; Accepted June 12, 2017

DOI: $10.3892 /$ or.2017.5753

\begin{abstract}
SIRT6, a member of NAD positivity-dependent class III deacetylase sirtuin family, played versatile roles in human cancers. However, the expression and biological function of SIRT6 in gastric cancer (GC) remain to be investigated. In this study, we found that SIRT6 expression level was decreased in gastric cancer tissues and cell lines. Decreased SIRT6 expression was associated with unfavorable clinical parameters including tumor differentiation, tumor size and TNM stage. Importantly, decreased level of SIRT6 was associated with decreased rate of overall survival (OS) and disease-free survival (DFS). Functionally, overexpression of SIRT6 in both BGC823 and SGC7901 cells inhibited cell viability and proliferation of GC cells. Furthermore, overexpression of SIRT6 in both BGC823 and SGC7901 cells prevented the cell cycle progress and increased cell apoptosis. In vivo experiments demonstrated that forced expression of SIRT6 in SGC-7901 cells inhibited the growth of SGC7901 cells in nude mice. Furthermore, the IHC staining for GC tissues showed that expression level of SIRT6 was decreased in GC tissues while the expression level of p-STAT3 was increased in GC tissues. GC tissues with high SIRT6 level showed significantly decreased level of p-STAT3. Mechanically, we demonstrated that SIRT6 blocked the activation of JAK2/STAT3 and inhibited the expression of cyclin D1 and $\mathrm{Bcl} 2$ which were downstream targets of JAK2/STAT3 pathway. Taken together, this study indicates that SIRT6 inhibits the growth of gastric cancer by inhibiting JAK2/STAT3 pathway.
\end{abstract}

\section{Introduction}

Gastric cancer (GC), with nearly 1 million new cases being diagnosed every year, is the second most common cause of

Correspondence to: Dr Jianwei Zhu, Department of Medical Oncology, Hangzhou Cancer Hospital, 34 Yan Guan Xiang, Shangcheng District, Hangzhou, Zhejiang 310002, P.R. China E-mail: zhu_jianweihz@163.com

Key words: SIRT6, gastric cancer, JAK2/STAT3 pathway, tumor growth cancer-related deaths (1). Although advancement in early diagnosis and therapeutic strategy has been achieved, the prognosis of GC patients remains poor $(2,3)$. The poor prognosis of $\mathrm{GC}$ patients is partly due to the fact that the molecular mechanisms underlying growth and metastasis of gastric cancer remain unknown.

SIRT6, belong to a conserved family of NAD positivitydependent enzymes, playing an important role in maintaining cellular and organismal homeostasis $(4,5)$. It was found to be involved in regulating mitochondrial oxidative metabolism and cellular glucose uptake $(6,7)$. It was also found to suppress obesity (8), cardiac hypertrophy (9), inflammation (10) and cellular senescence (11). In human cancers, SIRT6 was found to play different roles in different cancer types. SIRT6 has been reported to have both tumor suppressor and oncogenic properties. Reduced expression of SIRT6 has been found in colon cancer (12), hepatocellular carcinoma (13) and head and neck squamous cell carcinoma (14). Its decreased expression was correlated with advanced cancer stage and grade, and with poorer survival of cancer patients. In contrast, high SIRT6 levels were demonstrated in breast cancer (15) and prostate cancer (16), and were associated with the drug resistance and poor prognosis of cancer patients. However, the expression and biological function of SIRT6 in GC remains unknown.

In this study, we found that SIRT6 was significantly decreased in GC tissues and cell lines. Decreased expression of SIRT6 in GC patients was associated with poor clinicopathological features and worse prognosis. Functionally, SIRT6 inhibited cell viability, proliferation, and cell cycle progression while promoted apoptosis of GC cells. In vivo experiments showed that SIRT6 inhibited the growth of SGC7901 cells in nude mice. Moreover, we found that p-STAT3 expression was negatively correlated with SIRT6 expression in GC tissues. SIRT6 inhibited the JAK2/STAT3 pathway in GC cells.

\section{Materials and methods}

Cell cultures. Gastric cancer cell lines SGC-7901, MKN-45, BGC-823, and normal gastric epithelial GES-1 cells were obtained from ATCC (Rockville, MD, USA) and the Cell Bank of Chinese Academy of Sciences (Shanghai, China). These cells were cultured in RPMI-1640 medium (Life Technologies, Inc., Gaithersburg, MD, USA) along with $10 \%$ fetal bovine serum (Gibco Co., New York, NY, NY, USA), penicillin (100 U/ml), 
and streptomycin $(100 \mathrm{mg} / \mathrm{ml})$. All cell cultures were kept at $37^{\circ} \mathrm{C}$ in a humidified incubator with $5 \% \mathrm{CO}_{2}$.

Clinical tissues. GC tissues (68 pairs) and non-tumor tissues were collected from Hangzhou Cancer Hospital, and were stored at liquid nitrogen before using them for further experiments. Informed consents were obtained from all enrolled patients in this study. Demographic and clinicopathological information of the included patients are presented in Table I. Approval for carrying out the experiments in human tissue samples were obtained from the Institutional Research Ethics Committee of Hangzhou Cancer Hospital.

Cell transfection. Lentivirus encoding SIRT6 overexpressing vector and control vector were obtained from Genechem (Shanghai, China). Before virus transfection, GC cells were plated in 6-well plates, and the viruses diluted in complete medium $(2 \mathrm{ml})$ with polybrene $(8 \mu \mathrm{g} / \mathrm{ml})$ were added in the culture plates. Three days later, these cells were collected and western blotting was used to confirm the overexpression effect of the lentivirus.

Western blotting. Cellular proteins from GC cells were extracted with the RIPA lysis buffer and were quantified with a BCA protein assay kit (Pierce, Rockford, IL, USA), and $30 \mu \mathrm{g}$ cellular proteins were separated on $10 \%$ SDS-PAGE and transferred to NC membrane. Primary antibodies were incubated with the membranes overnight at $4^{\circ} \mathrm{C}$. The following antibodies were used in this study SIRT6 (1:1,000, Cell Signaling Technologies, Danvers, MA, USA), p-JAK2 (1:1,000, Cell Signaling Technologies), JAK (1:1,000, Cell Signaling Technologies), p-STAT3 (1:1000, Cell Signaling Technologies), STAT3 (1:1,000, Cell Signaling Technologies), cyclin D1 (1:1,500, Cell Signaling Technologies), Bcl-2 (1:500, Cell Signaling Technologies) and GAPDH (1:2,000, Santa Cruz, CA, USA). The membranes were incubated with secondary antibodies (1:3,000, Santa Cruz) at room temperature for $1 \mathrm{~h}$. The protein signals were visualized with ECL reagents (Amersham Biosciences Corp., USA). Western blots were semi-quantified by ImageJ software (1.46; National Institutes of Health, Bethesda, MD, USA).

Cell viability and proliferation assay. MTT assay was used for measuring cell viability. In brief, 5,000 GC cells were plated into 96-well plates. These cells were stained with MTT (Sigma, St. Louis, MO, USA) for $4 \mathrm{~h}$ at $37^{\circ} \mathrm{C}$ at corresponding time points $(0,24,48$ and $72 \mathrm{~h})$, and the absorbance at $490 \mathrm{~nm}$ was measured to reflect the cell viability. For cell proliferation, colony formation assay was performed. GC cells transfected with control vector or SIRT6 overexpressing vector were seeded in 6-well plates and maintained in cell incubators for 10 days. The formed cell colonies were stained with crystal violet solution. The number of cell colonies was counted to represent the cell proliferation ability of GC cells.

IHC staining. GC tissues and the adjacent non-tumor tissues were subjected to formalin fixation and were embedded with paraffin, $5 \mu \mathrm{m}$ thick tissue sections were used for IHC staining following the standard IHC protocol. Staining intensity was scored as no 0 , staining; 1 , weak staining; 2 , moderate
Table I. The correlation between SIRT6 expression and clinicopathological features in gastric cancer.

\begin{tabular}{|c|c|c|c|c|}
\hline \multirow[b]{2}{*}{ Characteristics } & \multirow[b]{2}{*}{ Total } & \multicolumn{2}{|c|}{ SIRT6 expression } & \multirow[b]{2}{*}{ P-value } \\
\hline & & $\begin{array}{r}\text { Low } \\
(52)\end{array}$ & $\begin{array}{l}\text { High } \\
(16)\end{array}$ & \\
\hline \multicolumn{5}{|l|}{ Age (years) } \\
\hline$<65$ & 30 & 21 & 9 & 0.264 \\
\hline$\geq 65$ & 38 & 31 & 7 & \\
\hline \multicolumn{5}{|l|}{ Sex } \\
\hline Male & 52 & 42 & 10 & 0.132 \\
\hline Female & 16 & 10 & 6 & \\
\hline \multicolumn{5}{|l|}{ Tumor differentiation } \\
\hline $\mathrm{I}, \mathrm{II}$ & 31 & 19 & 12 & $0.007^{\mathrm{a}}$ \\
\hline III, IV & 37 & 33 & 4 & \\
\hline \multicolumn{5}{|l|}{ Size $(\mathrm{cm})$} \\
\hline$<5$ & 30 & 19 & 11 & $0.023^{\mathrm{a}}$ \\
\hline$\geq 5$ & 38 & 33 & 5 & \\
\hline \multicolumn{5}{|l|}{ Invasive depth } \\
\hline $\begin{array}{l}\text { Mucosa to muscularis } \\
\text { propria }\end{array}$ & 10 & 5 & 5 & 0.083 \\
\hline $\begin{array}{l}\text { Adventitia to adjacent } \\
\text { structure }\end{array}$ & 58 & 47 & 11 & \\
\hline \multicolumn{5}{|l|}{ Lymph node metastasis } \\
\hline$\leq 2$ regions & 31 & 23 & 8 & 0.685 \\
\hline$>2$ regions & 37 & 29 & 8 & \\
\hline \multicolumn{5}{|l|}{ Distant metastasis } \\
\hline No & 54 & 44 & 10 & 0.119 \\
\hline Yes & 14 & 8 & 6 & \\
\hline \multicolumn{5}{|l|}{ Venous infiltration } \\
\hline Absent & 50 & 39 & 11 & 0.864 \\
\hline Present & 18 & 13 & 5 & \\
\hline \multicolumn{5}{|l|}{ TNM stage } \\
\hline I, II & 29 & 18 & 11 & $0.016^{\mathrm{a}}$ \\
\hline III, IV & 39 & 34 & 5 & \\
\hline
\end{tabular}

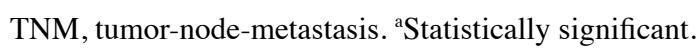

staining; and 3, strong staining. Staining quantity was graded as $1,<25 \% ; 2,25-75 \%$; and $3,>75 \%$. IHC score was manually confirmed by two independent experienced pathologists using the formula: IHC score = staining intensity x staining quantity.

Cell cycle and apoptosis analysis. For cell cycle assay, GC cells were collected $72 \mathrm{~h}$ after virus transfection. These cells were fixed with $80 \%$ ethanol overnight, and then were stained with propidium iodide $(50 \mu \mathrm{g} / \mathrm{ml}$, BD Biosciences, Franklin Lakes, NJ, USA) at room temperature for $30 \mathrm{~min}$. The percentage of cells in each cell cycle was measured with FACSCalibur system (BD Biosciences). For apoptosis assay, 
A

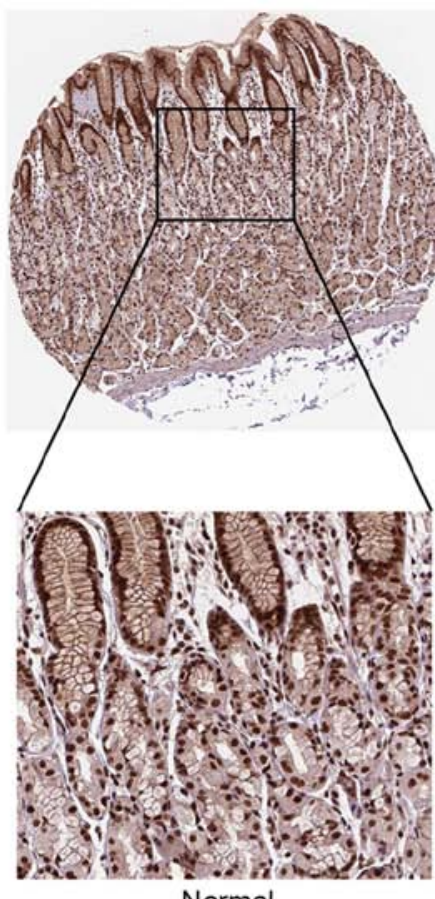

Normal

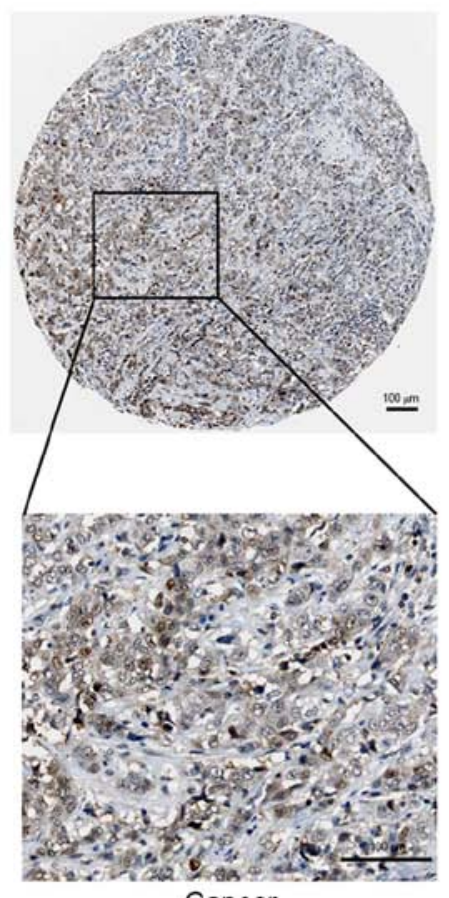

Cancer
B

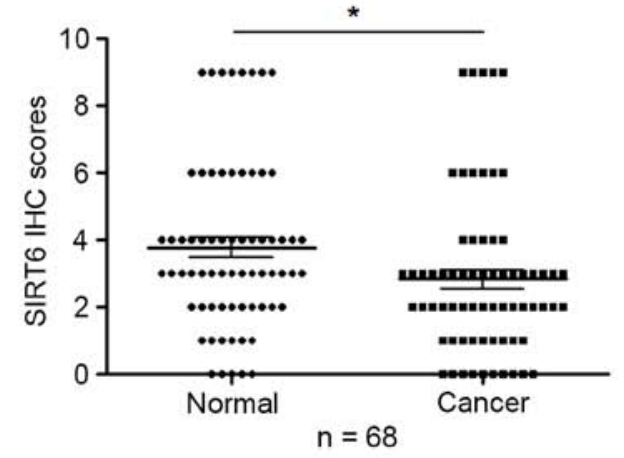

C
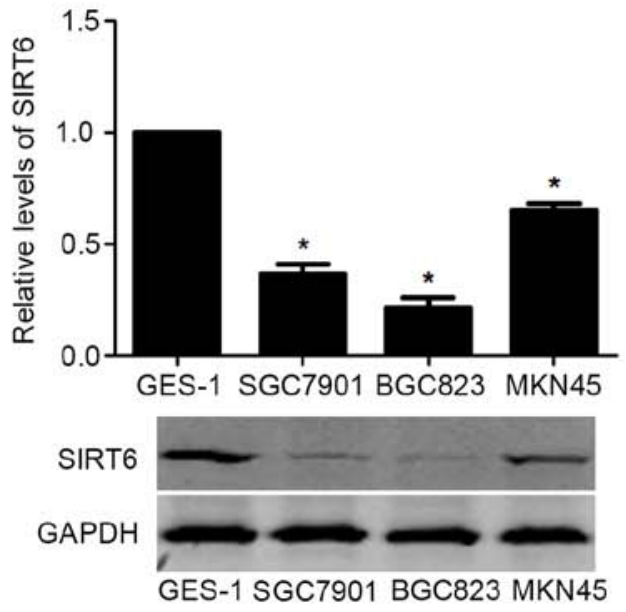

Figure 1. The expression of SIRT6 was decreased in gastric cancer. (A) Representative IHC staining results for SIRT6 in gastric cancer tissues and matched adjacent normal tissues are presented. (B) The expression level of SIRT6 was decreased in GC tissues compared with that in non-tumor tissues. $n=68$, ${ }^{*} \mathrm{P}<0.05$ by t-test. (C) Relative expression level of SIRT6 in gastric cancer cell lines and GES-1 cells. The expression of SIRT6 was significantly decreased in GC cell lines (SGC7901, BGC823 and MKN45) compared with that in GES-1 cells. $\mathrm{n}=3$ independent experiments, ${ }^{*} \mathrm{P}<0.05$ by ANOVA.

GC cells after transfection were subjected to an apoptosis assay. The percentage of apoptotic GC cells were measured using Annexin V/propidium iodide kit (BD Pharmingen, San Diego, CA, USA) according to the manufacturer's instructions.

In vivo tumor growth assay. For tumor growth studies, nude mice were injected subcutaneously with $1 \times 10^{6}$ SGC7901 cells transfected with control vector or SIRT6 vector. Tumor sizes were measured every 3 days after subcutaneous injection. Three weeks later, the mice were sacrificed by cervical dislocation under anesthesia, and tumors were removed for the volume measurement. The protocols for animal experiments were approved by the Animal Care Committee of Hangzhou Cancer Hospital.

Statistical analysis. All quantitative data in this study are presented as mean \pm standard error of the mean (SEM). Statistical analysis including Student's t-test, ANOVA analysis, Chi-square test, Correlation analysis, and Kaplan-Meier analysis was performed with Graphpad software. $\mathrm{P}<0.05$ was considered as statistically significant.

\section{Results}

SIRT6 expression is decreased in GC tissues and cells. To investigate the expression level of SIRT6 in GC, we performed
IHC staining of SIRT6 in GC tissues. IHC staining showed that SIRT6 expression was significantly lower in GC tissues compared with that in adjacent non-tumor tissues (Fig. 1A) compared with that in adjacent non-tumor tissues. IHC scoring for SIRT6 in GC confirmed that the expression level of SIRT6 in GC tissues was significantly increased $(\mathrm{P}<0.05$, Fig. 1B). Moreover, we investigated the expression level of SIRT6 in GC cells lines and GES-1 cells. Compared with that in GES-1 cells, the protein level of SIRT6 was significantly decreased in GC cell lines including SGC7901, BGC823 and MKN45 cells $(\mathrm{P}<0.05$, Fig. 1C).

Decreased SIRT6 expression in GC tissues is associated with poor clinicopathological features and prognosis of $G C$ patients. After confirming the decreased expression of SIRT6 expression in GC, we further determined whether decreased SIRT6 expression level was correlated with the clinical features and the prognosis of GC patients. GC patients were divided into two groups according to the IHC scores: SIRT6 low expression group (IHC score <4) and SIRT6 high expression group (IHC score $\geq 4$ ). Clinical association analysis showed that patients with lower SIRT6 level had lower grade of tumor differentiation, large tumor size and advanced TNM stage $(\mathrm{P}<0.05$, respectively). More importantly, decreased level of SIRT6 was associated with decreased rate of overall survival ( $\mathrm{P}=0.002$, Fig. 2$)$ and disease-free survival ( $\mathrm{P}=0.007$, Fig. 2). 

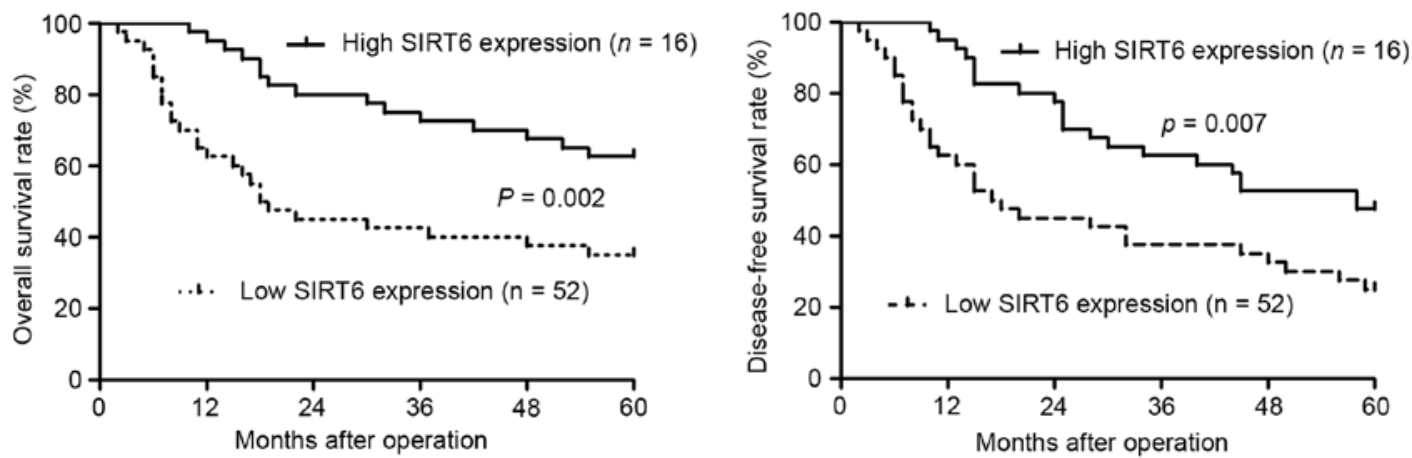

Figure 2. Decreased SIRT6 expression is correlated with poor prognosis of GC patients. Overall survival (OS) and disease-free survival (DFS) was compared between patients with high level of SIRT6 $(n=16)$ and those with low SIRT6 level $(n=52)$. Decreased SIRT6 expression is correlated with decreased rate of OS and DFS in GC patients. $\mathrm{P}<0.05$ by log-rank test.

A

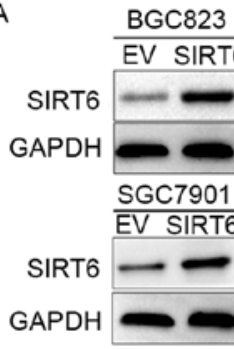

B

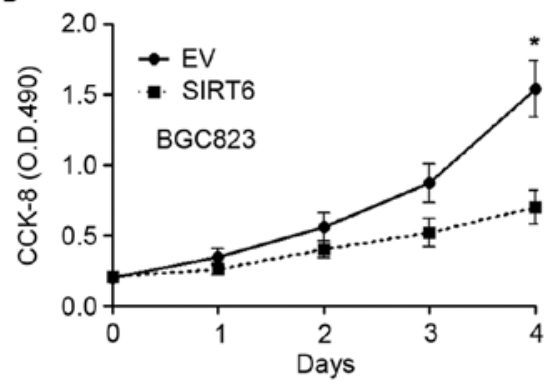

C
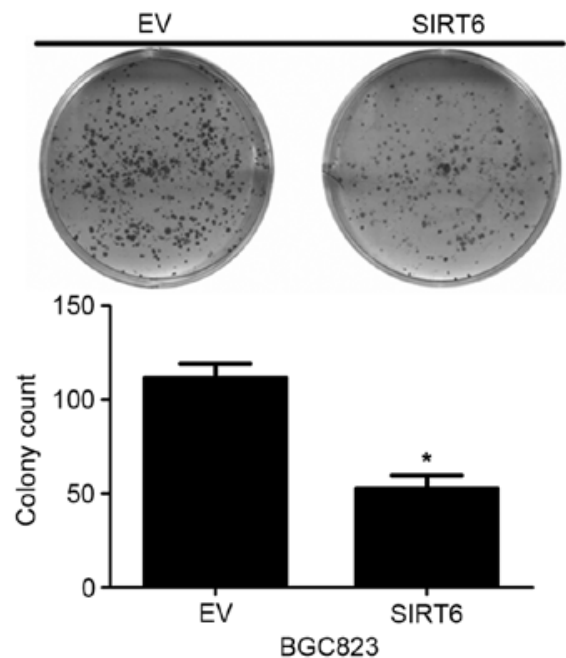
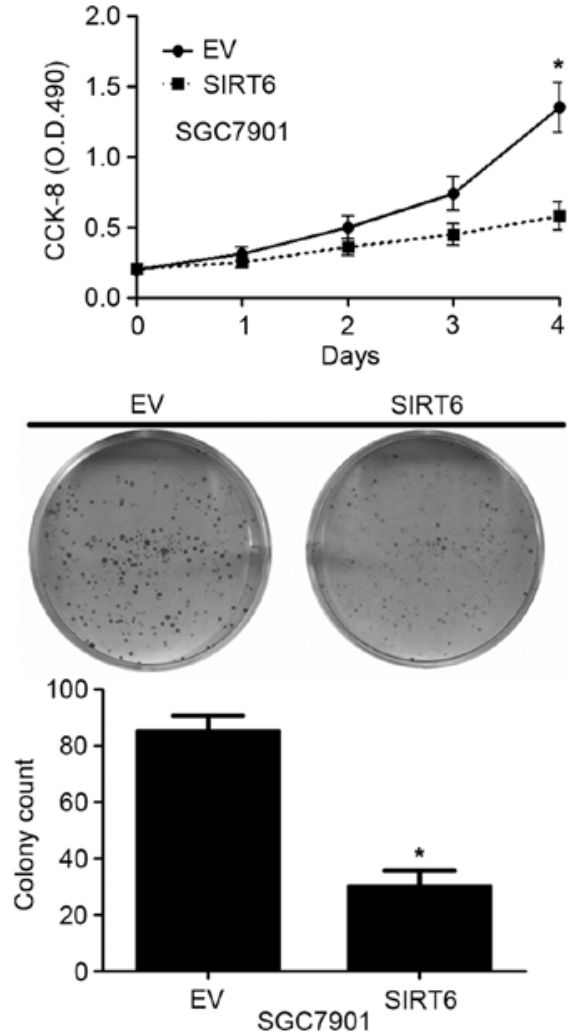

Figure 3. Overexpression of SIRT6 inhibits cell viability and proliferation of GC cells. (A) Lentivirus encoding SIRT6 vector significantly increased SIRT6 expression in BGC823 and SGC7901 cells. (B) MTT assay was performed to evaluate cell viability of GC cells. SIRT6 overexpression significantly decreased cell viability of BGC823 and SGC7901 cells. $\mathrm{n}=3$ independent experiments, ${ }^{*} \mathrm{P}<0.05$ by ANOVA. (C) Colony formation assay was performed to evaluate cell proliferation of GC cells. SIRT6 overexpression significantly decreased cell proliferation of BGC 823 and SGC7901 cells. $n=3$ independent experiments, ${ }^{*} \mathrm{P}<0.05$ by t-test.

SIRT6 inhibits cell viability and proliferation of GC cells. Then, we investigated the biological function of SIRT6 in GC cells. Lentivirus encoding SIRT6 overexpressing vector significantly increased SIRT6 expression in BGC823 and SGC7901 cells as suggested by western blotting (Fig. 3A). MMT assay showed that forced expression of SIRT6 inhibited the cell viability of BGC823 and SGC7901 cells ( $\mathrm{P}<0.05$, Fig. 3B). Moreover, colony formation assay showed that overexpressing SIRT6 significantly inhibited the colony formation of BGC823 and SGC7901 cells ( $\mathrm{P}<0.05$, Fig. 3C). These data indicate SIRT6 inhibited the cell viability and proliferation of GC cells.
SIRT6 inhibits cell cycle progression and increases apoptosis of GC cells. Then, we investigated whether SIRT6 could influence cell cycle progression and apoptosis of GC cells. SIRT6 overexpression in both BGC823 and SGC7901 cells increased the percentage of GC cells in G1 stage while decreased the percentage of GC cells in $\mathrm{S}$ phase $(\mathrm{P}<0.05$, Fig. $4 \mathrm{~A})$. On the other hand, overexpression of SIRT6 increased the percentage of apoptotic BGC823 and SGC7901 cells ( $\mathrm{P}<0.05$, Fig. 4B).

SIRT6 inhibits the growth of SGC7901 cells in nude mice. To further investigate whether SIRT6 affects the in vivo growth 
A
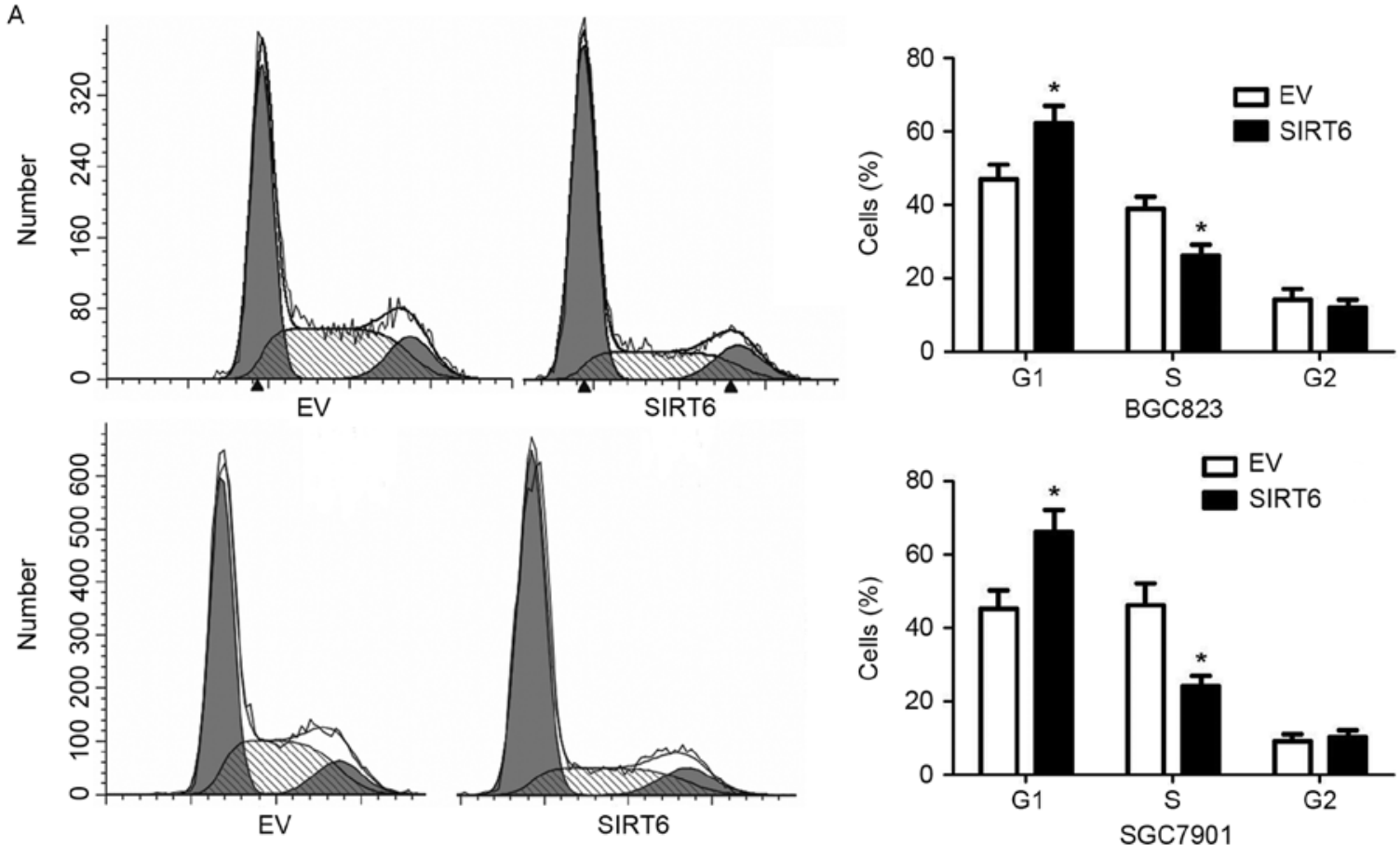

B
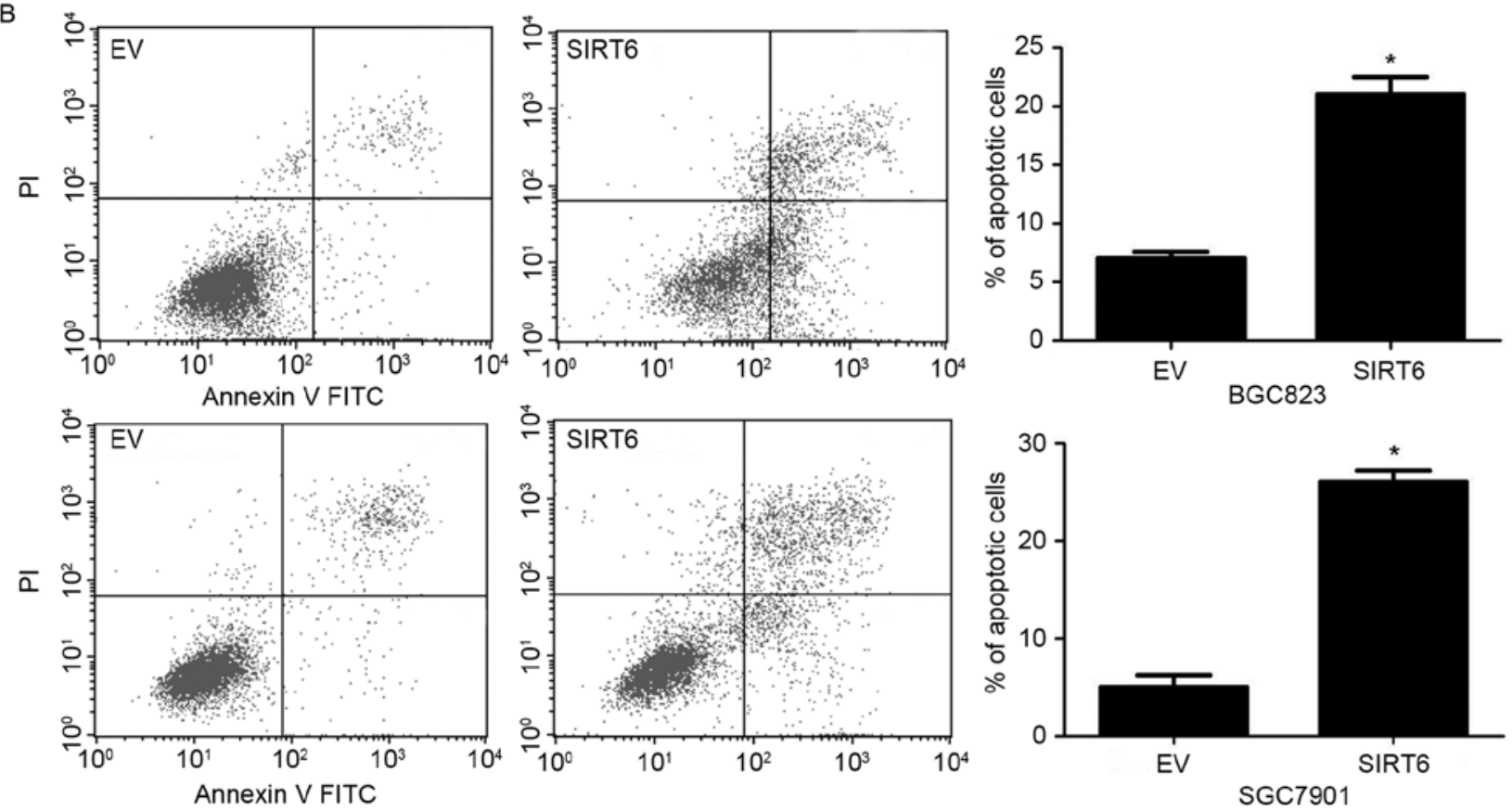

Figure 4. Overexpression of SIRT6 inhibits cell cycle progression and enhanced apoptosis of GC cells. Flow cytometry analysis was performed to evaluate the cell cycle progression and apoptosis of GC cells. (A) SIRT6 overexpression significantly increased the percentage of BGC823 and SGC7901 cells in G1 phase and decreased the percentage of cells in S phase, $n=3$ independent experiments, " $\mathrm{P}<0.05$ by $\mathrm{t}$-test. (B) SIRT6 overexpression significantly increased the percentage of apoptotic BGC823 and SGC7901 cells, $\mathrm{n}=3$ independent experiments, ${ }^{*} \mathrm{P}<0.05$ by t-test.

of GC cells, we carried out subcutaneous injection experiment with SGC7901. The result of subcutaneous tumor formation showed that the in vivo growth of SGC7901 cells was significantly inhibited after overexpression of SURT6 (Fig.5, $\mathrm{P}<0.05)$. These data suggest that SIRT6 inhibited the growth of GC cells in vivo.

SIRT6 inhibits JAK2/STAT3 signaling pathway in GC cells. Since JAK2/STAT3 signaling pathway has been confirmed to be a critical pathway in regulating the growth of GC cells, we investigated whether SIRT6 could inhibit JAK2/ STAT3 pathway in GC cells. To answer this question, we used IHC staining to determine whether the expression of p-STAT3, which was marker of JAK2/STAT3 pathway, was negatively correlated with SIRT6 expression in GC tissues. Serial sectioning of GC tissues showed that in adjacent nontumor tissue with high SIRT6 expression, the expression of p-STAT3 was low (Fig. 6A, upper part). In tumor tissues with low SIRT6 expression, the expression of p-STAT3 was high (Fig. 6A, lower part). The result of p-STAT3 IHC score 

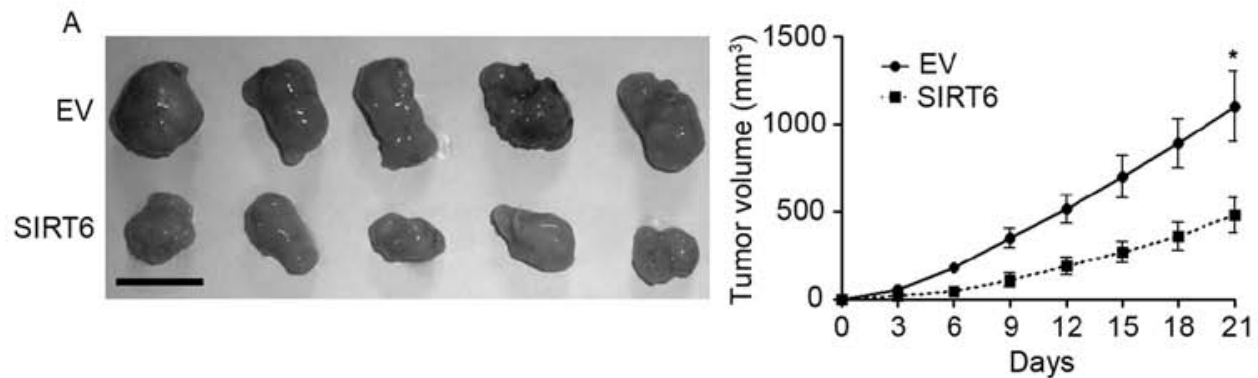

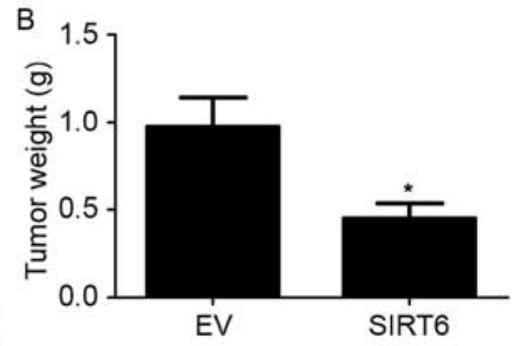

Figure 5. Overexpression of SIRT6 inhibits the growth of SGC7901 cells in nude mice. (A) Overexpression of SIRT6 significantly slowed down the tumor growth of SGC7901 cells in nude mice. $n=5,{ }^{*} \mathrm{P}<0.05$ by ANOVA. (B) Tumor volumes were significantly decreased in nude mice injected with SGC7901 cells overexpressing SIRT6. $\mathrm{n}=5,{ }^{*} \mathrm{P}<0.05$ by t-test.

A
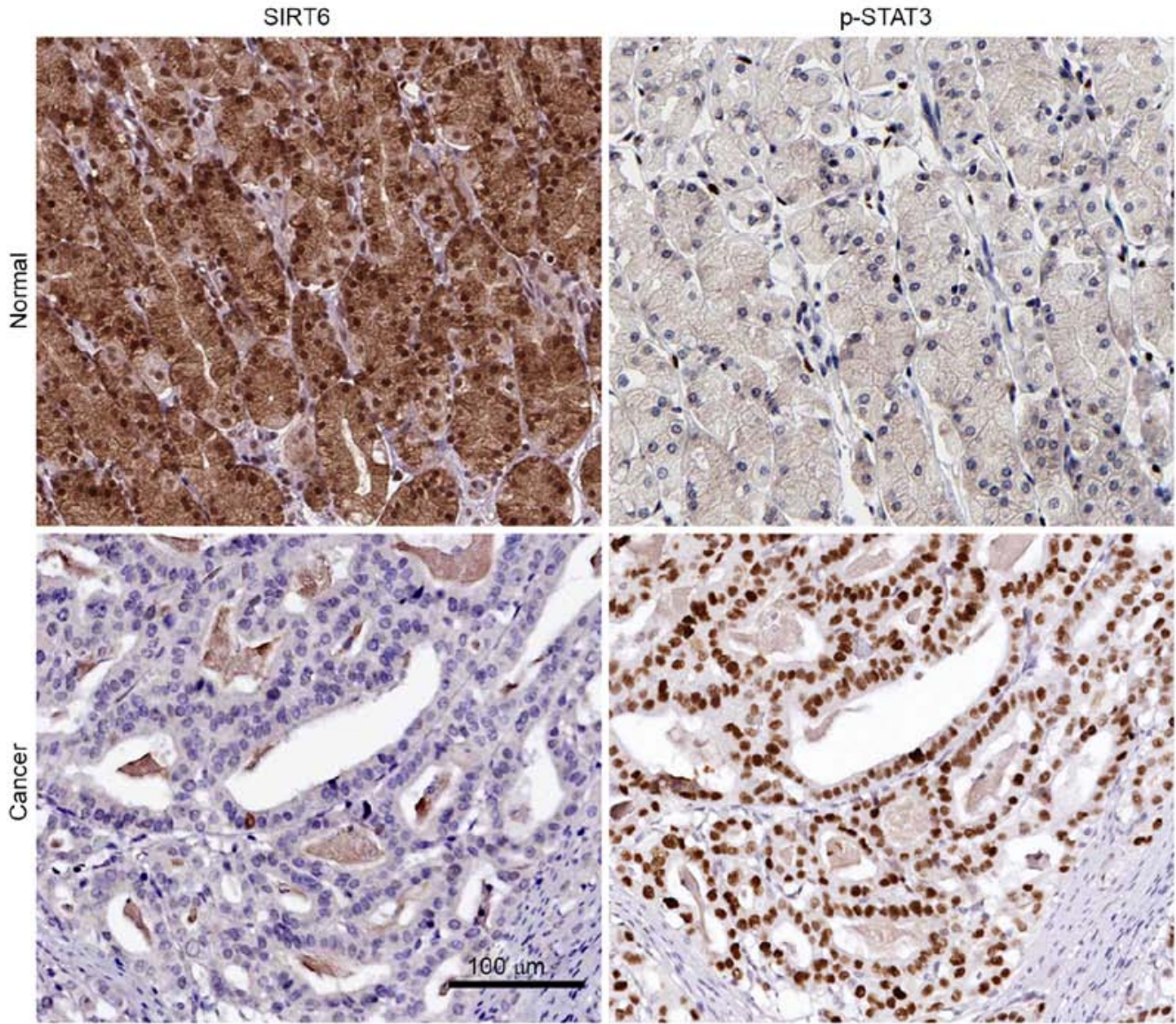

B

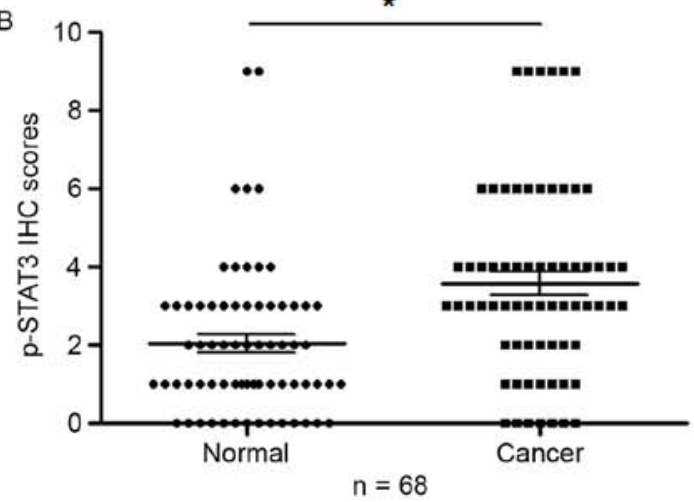

C *

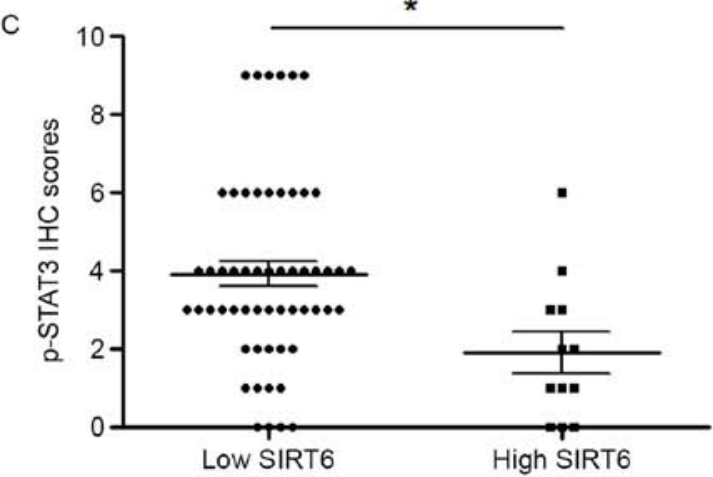

Figure 6. The expression of p-STAT3 is negatively correlated with SIRT6 expression in GC tissues. (A) Serial section staining for p-STAT3 and SIRT6 in GC tissues and non-tumor tissue. Non-tumor tissues with high expression of SIRT6 showed low or negative expression of p-STAT3. GC tissues with low expression of SIRT6 showed increased expression of p-STAT3. (B) The expression level of p-STAT3 was significantly increased in GC tissues compared with non-tumor tissues. $\mathrm{n}=68,{ }^{*} \mathrm{P}<0.05$ by t-test. (C) GC tissues with high SIRT6 expression ( $\mathrm{n}=16$ ) showed significantly decreased expression level of $\mathrm{p}$-STAT3 compared to SIRT6 low expressing cases $(\mathrm{n}=52)$. ${ }^{*} \mathrm{P}<0.05$ by t-test. 


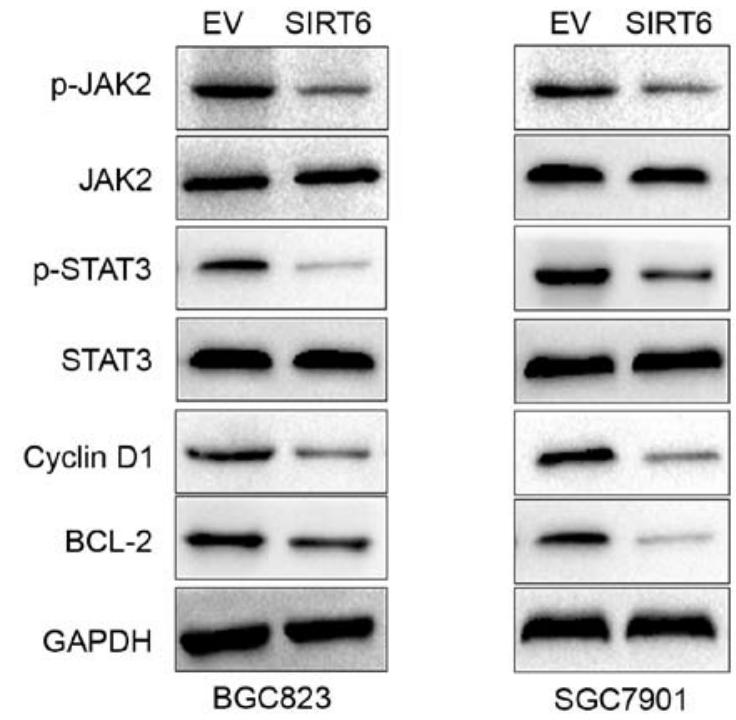

Figure 7. SIRT6 overexpression inhibits JAK2/STAT3 pathway in GC cells Western blotting demonstrated that SIRT6 overexpression significantly decreased p-JAK2, p-STAT3, cyclin D1 and Bcl-2 expression in BGC823 and SGC7901 cells.

showed that the expression of p-STAT3 was significantly increased in GC tissues $(\mathrm{P}<0.05$, Fig. 6B). Importantly, in tissues with low SIRT6 level, the expression of p-STAT3 was significantly higher than that in tissues with high SIRT6 level $(\mathrm{P}<0.05$, Fig. 6C). To further confirm SIRT6 could affect the JAK2/STAT3 pathway in GC cells, we used western blotting to investigate the expression of p-JAK2, JAK2, p-STAT3 and STAT3, and Bcl-2 and cyclin D1 which are downstream markers of the activation of JAK2/STAT3 pathway, after SIRT6 overexpression. Overexpression of SIRT6 in BGC823 and SGC7901 cells significantly reduced the level of p-JAK2, p-STAT3, cyclin D1 and Bcl-2 (Fig. 7). These data indicate that SIRT6 inhibited JAK2/STAT3 pathway in GC cells.

\section{Discussion}

SIRT6 was found to be a versatile protein which was involved in various biological processes including mitochondrial oxidative metabolism (6), cellular glucose uptake (7), inflammation (10), cardiac hypertrophy (9), DNA repair (17), and resistance to heat stress (18). Studies on cancer showed that SIRT6 could either act as tumor suppressors or as oncogenic protein in different types of human cancers. However, the expression and function of SIRT6 in GC were not previously investigated. In this study, we showed that the expression of SIRT6 was significantly decreased in GC tissues and cell lines. Association analysis further confirmed that decreased expression of SIRT6 was associated with unfavorable clinical features and poor prognosis of GC patients indicating that SIRT6 played tumor suppressive role in GC.

Previously, SIRT6 expression was also found to be decreased in colon (12), liver (13), and head and neck squamous cell carcinomas (14). Functionally, SIRT6 was found to play tumor suppressive roles by inhibiting the cell proliferation, lipogenesis and glycosis and enhancing apoptosis (19). In this study, in vitro assays showed that SIRT6 could inhibit cell viability, proliferation and cell cycle progression while promoted the apoptosis of GC cells. In vivo experiments further confirmed that SIRT6 suppressed the in vivo growth of SGC7901 cells in nude mice. These date indicate SIRT6 played tumor suppressive role in GC cells by inhibiting tumor growth.

Previous studies showed that SIRT6 could regulate p53 and p73 apoptosis pathways (20), HIF-1 $\alpha$ pathway (21) and insulinIGF-1-like signaling pathway (22). JAK2/STAT3 signaling pathway is a well-characterized pathway which promotes the growth of GC cells (23). Since we found SIRT6 inhibits the growth of GC cells, we further investigated whether SIRT6 could inhibit the growth of GC cells by inhibiting JAK2/ STAT3 pathway. The results of IHC staining in GC tissues and western blotting in GC cells demonstrated that SIRT6 inhibited JAK2/STAT2 pathway activation in GC. The above suggest that SIRT6 inhibited the growth of GC cells by inhibiting the JAK2/STAT3 pathway.

In conclusion, we demonstrated that SIRT6 expression was decreased in GC tissues and cells. Decreased expression level of SIRT6 was associated with poor clinical features and prognosis of GC patients. SIRT6 inhibited the cell viability, proliferation and cell cycle progression and increased apoptosis of GC cells. In vivo experiments confirmed that SIRT6 suppressed the growth of SGC7901 cells in nude mice. Mechanically, we demonstrated that SIRT6 inhibited JAK2/ STAT3 pathway activation in GC cells. Therefore, this study indicates that SIRT6 is a promising biomarker and therapeutic target for GC patients.

\section{References}

1. Crew KD and Neugut AI: Epidemiology of gastric cancer. World J Gastroenterol 12: 354-362, 2006.

2. Saragoni L, Morgagni P, Gardini A, Marfisi C, Vittimberga G, Garcea D and Scarpi E: Early gastric cancer: Diagnosis, staging, and clinical impact. Evaluation of 530 patients. New elements for an updated definition and classification. Gastric Cancer 16: 549-554, 2013.

3. Carcas LP: Gastric cancer review. J Carcinog 13: 14, 2014.

4. Gertler AA and Cohen HY: SIRT6, a protein with many faces. Biogerontology 14: 629-639, 2013.

5. Pan PW, Feldman JL, Devries MK, Dong A, Edwards AM and Denu JM: Structure and biochemical functions of SIRT6. J Biol Chem 286: 14575-14587, 2011.

6. Houtkooper RH, Pirinen E and Auwerx J: Sirtuins as regulators of metabolism and healthspan. Nat Rev Mol Cell Biol 13: 225-238, 2012.

7. Xiao C, Kim H-S, Lahusen T, Wang RH, Xu X, Gavrilova O, Jou W, Gius D and Deng CX: SIRT6 deficiency results in severe hypoglycemia by enhancing both basal and insulin-stimulated glucose uptake in mice. J Biol Chem 285: 36776-36784, 2010.

8. Kanfi Y, Peshti V, Gil R, Naiman S, Nahum L, Levin E, KronfeldSchor N and Cohen HY: SIRT6 protects against pathological damage caused by diet-induced obesity. Aging Cell 9: 162-173, 2010.

9. Sundaresan NR, Vasudevan P, Zhong L, Kim G, Samant S, Parekh V, Pillai VB, Ravindra PV, Gupta M, Jeevanandam V, et al: The sirtuin SIRT6 blocks IGF-Akt signaling and development of cardiac hypertrophy by targeting c-Jun. Nat Med 18: 1643-1650, 2012.

10. Xiao C, Wang R-H, Lahusen TJ, Park O, Bertola A, Maruyama T, Reynolds D, Chen Q, Xu X, Young HA, et al: Progression of chronic liver inflammation and fibrosis driven by activation of c-JUN signaling in Sirt6 mutant mice. J Biol Chem 287: 41903-41913, 2012.

11. Mao Z, Tian X, Van Meter M, Ke Z, Gorbunova V and Seluanov A: Sirtuin 6 (SIRT6) rescues the decline of homologous recombination repair during replicative senescence. Proc Natl Acad Sci USA 109: 11800-11805, 2012. 
12. Sebastián C, Zwaans BM, Silberman DM, Gymrek M, Goren A Zhong L, Ram O, Truelove J, Guimaraes AR, Toiber D, et al: The histone deacetylase SIRT6 is a tumor suppressor that controls cancer metabolism. Cell 151: 1185-1199, 2012.

13. Zhang Z-G and Qin C-Y: Sirt6 suppresses hepatocellular carcinoma cell growth via inhibiting the extracellular signal-regulated kinase signaling pathway. Mol Med Rep 9: 882-888, 2014.

14. Lu C-T, Hsu C-M, Lin P-M, Lai CC, Lin HC, Yang CH, Hsiao HH, Liu YC, Lin HY, Lin SF, et al: The potential of SIRT6 and SIRT7 as circulating markers for head and neck squamous cell carcinoma. Anticancer Res 34: 7137-7143, 2014.

15. Khongkow M, Olmos Y, Gong C, Gomes AR, Monteiro LJ, Yagüe E, Cavaco TB, Khongkow P, Man EP, Laohasinnarong S, et al: SIRT6 modulates paclitaxel and epirubicin resistance and survival in breast cancer. Carcinogenesis 34: 1476-1486, 2013.

16. Liu Y, Xie QR, Wang B, Shao J, Zhang T, Liu T, Huang G and $\mathrm{Xia} W$ : Inhibition of SIRT6 in prostate cancer reduces cell viability and increases sensitivity to chemotherapeutics. Protein Cell 4: 702-710, 2013

17. Mao Z, Hine C, Tian X, Van Meter M, Au M, Vaidya A, Seluanov A and Gorbunova V: SIRT6 promotes DNA repair under stress by activating PARP1. Science 332: 1443-1446, 2011.
18. Chiang W-C, Tishkoff DX, Yang B, Wilson-Grady J, Yu X, Mazer T, Eckersdorff M, Gygi SP, Lombard DB and Hsu AL: C. elegans SIRT6/7 homolog SIR-2.4 promotes DAF-16 relocalization and function during stress. PLoS Genet 8: e1002948, 2012.

19. Roth M and Chen WY: Sorting out functions of sirtuins in cancer. Oncogene 33: 1609-1620, 2014.

20. Van Meter M, Mao Z, Gorbunova V and Seluanov A: SIRT6 overexpression induces massive apoptosis in cancer cells but not in normal cells. Cell Cycle 10: 3153-3158, 2011.

21. Barneda-Zahonero B and Parra M: Histone deacetylases and cancer. Mol Oncol 6: 579-589, 2012.

22. Leiser SF, Fletcher M, Begun A and Kaeberlein M: Life-span extension from hypoxia in Caenorhabditis elegans requires both HIF-1 and DAF-16 and is antagonized by SKN-1. J Gerontol A Biol Sci Med Sci 68: 1135-1144, 2013.

23. Judd LM, Menheniott TR, Ling H, Jackson CB, Howlett M, Kalantzis A, Priebe W and Giraud AS: Inhibition of the JAK2/ STAT3 pathway reduces gastric cancer growth in vitro and in vivo. PLoS One 9: e95993, 2014. 